\title{
Migración Inclusiva en Chile, un Desafío Educativo Vigente
}

\section{Inclusive Migration in Chile, an Ongoing Educational Challenge}

\author{
David Román Soto * \\ Universidad Metropolitana de Ciencias de la Educación, Chile
}

\begin{abstract}
El presente artículo tiene como objetivo reflexionar en torno a la migración en Chile, sus características y aproximaciones conceptuales y estadísticas que permitan la comprensión del fenómeno en la actualidad cuando se observa desde la vulnerabilidad social, considerando las consecuencias que ésta acarrea para la población migrante cuando intentan ser incluidos en un país extranjero. Se abordará la temática desde lo que ocurre con la política pública vigente en relación a la migración y cómo ha permeado el desarrollo de la escuela y las reflexiones que de ella se desprenden. Acto seguido, se presentarán algunas investigaciones que dan cuenta de la manera en que se aborda el fenómeno de la migración al interior de contextos escolares formales y su repercusión en el desarrollo de procesos educativos inclusivos y de aprendizaje. Finalmente, a partir de un enfoque poscrítico, tanto la educación inclusiva como la interculturalidad nos brindarán elementos que permitan dilucidar respuestas a los requerimientos y necesidades propias de la población migrante, en torno a la transformación que debe realizar la escuela en tanto institución, y en particular al interior del espacio didáctico, a fin de considerar a la diversidad del estudiantado como principio fundamental.
\end{abstract}

Descriptores: Migración; Vulnerabilidad; Inclusión; Diversidad; Interculturalidad.

The purpose of this article is to reflect on migration in Chile, its characteristics and conceptual and statistical approaches that allow the understanding of the current phenomenon when it is observed from the perspective of social vulnerability, considering the consequences it entails for the migrant population when they try to be included in a foreign country. The subject will be approached from what happens with the current public policy in relation to migration and how it has permeated the development of the school and the reflections that arise from it. Then, some researches will be presented that show the way in which the migration phenomenon is approached within formal school contexts and its impact on the development of inclusive educational and learning processes. Finally, from a post-critical approach, both inclusive education and interculturality will provide us with elements that will allow us to elucidate answers to the requirements and needs of the migrant population, regarding the transformation that the school must carry out as an institution, and in particular within the didactic space, in order to consider the diversity of the student body as a fundamental principle.

Keywords: Migration; Vulnerability; Inclusion; Diversity; Interculturality. 


\section{Introducción}

En la actualidad, el fenómeno de la migración ha crecido aceleradamente en los últimos años. A nivel mundial, la población migrante asciende a 272 millones, 52 millones más que en el 2010 (ONU, 2019), lo cual ha provocado problemáticas de inclusión para los países de acogida, en torno a temas concernientes a vivienda, salud, trabajo y educación. Nuestro país no ha estado ajeno al incremento de movilidad de la población, puesto que durante la última década se han suscitado cambios políticos, económicos y sociales en diversos países de la región, dada las dificultades de hombres y mujeres de poseer una vida plena y próspera en sus países de origen y/o residencia, lo que los hace buscar nuevos horizontes y perspectivas con el fin de mejorar su calidad de vida y la de sus familias.

En particular, la inclusión de estudiantes migrantes en el sistema educativo regular que recibe fondos públicos es una problemática que Chile está experimentando de forma acelerada y constante. Según el Ministerio de Educación, las cifras se han duplicado en los últimos años (MINEDUC, 2018), concentrándose en su mayoría en establecimientos de educación municipal (escuela pública) y de educación particular subvencionada (90,6\%). En ese sentido, la política de Inclusión Educativa dejó de estar focalizada en niños, niñas y jóvenes que presentan necesidades educativas especiales (NEE), sino que enfoca su accionar bajo el principio de diversidad con un Enfoque de Derechos, de Género y de Interculturalidad (MINEDUC, 2018), con la finalidad de brindar oportunidades reales y concretas de insertarse y permanecer en el sistema educativo a diversos grupos de la población sin realizar ninguna distinción de tipo arbitraria.

Para tales efectos, en el año 2015 se promulga la Ley 20.845 de Inclusión Escolar (Ley de Inclusión Escolar, 2015), la cual tiene como propósito la prohibición del lucro en establecimientos educacionales que reciben financiamiento del Estado, eliminar el financiamiento compartido (copago) y regular la admisión de los y las estudiantes, eliminando la selección del estudiantado por parte de los propios establecimientos educacionales, los cuales son puntos centrales en la segregación escolar que se había consolidado en los últimos años.

Sin embargo, una nueva arista surge cuando la población extranjera que migra con la finalidad de mejorar sus condiciones y su calidad de vida lo realiza hacia contextos vulnerables del país de acogida, puesto que sus redes de contacto y apoyo son escasas o nulas, y las posibilidades de una inclusión efectiva se tornan aún más dificultosas (Castel, 2009). Es ahí donde la escuela cumple un rol fundamental en este proceso, y no solo para los niños, niñas y jóvenes que pretenden participar del sistema regular de enseñanza, sino también para las familias del estudiante migrante. En coherencia con el Enfoque Inclusivo, el Enfoque de Interculturalidad (UNESCO, 2006), nace como respuesta a las nuevas miradas que se desprenden del fenómeno multicultural que se está haciendo presente en los establecimientos educacionales, con el fin de permitir una comprensión mayor del fenómeno de la migración desde una nueva racionalidad Poscrítica (Aranda, 2017).

En ese contexto, esta reflexión crítica se preocupará del fenómeno migratorio desde un punto de vista conceptual y epistemológico, en relación a lo que acontece en Chile en la actualidad, conceptualizando a la diversidad como eje transversal del proceso de inclusión que debe vivir la población migrante desde una concepción intercultural. Para tales efectos, en primer lugar, se abordarán los datos actualizados en torno a la migración en Chile y sus consecuencias en relación a la inclusión de migrantes en contextos de 
vulnerabilidad. Posteriormente se profundizará acerca de la política pública vigente de educación inclusiva, la cual promueve procesos inclusivos sin distinción, ahondando desde diferentes perspectivas que permitan entender la diversidad y sus consecuencias en el espacio didáctico. En un tercer apartado se abordarán ideas y concepciones de la interculturalidad en tanto enfoque que permite realizar prácticas socioeducativas coherentes y adecuadas a la Educación Inclusiva, las cuales en conjunto serán consideradas el motor central que nos permita reconceptualizar la práctica pedagógica en contextos de vulnerabilidad para la construcción de una nueva escuela desde una mirada intercultural.

\section{Migración y vulnerabilidad}

Según la Organización de Naciones Unidas, se comprende migración como "el movimiento de personas fuera de su hogar habitual, ya sea a través de una frontera internacional, o dentro de un país" (OIM, 2020). Así también define a un migrante como cualquier persona que se desplaza o se ha desplazado a través de una frontera internacional o dentro de un país, fuera de su lugar habitual de residencia independientemente de: 1) su situación jurídica; 2) el carácter voluntario o involuntario del desplazamiento; 3) las causas del desplazamiento; o 4) la duración de su estancia (IOM, 2020). La Declaración de Nueva York sobre Refugiados y Migrantes adoptada en 2016 define migrante como "alguien que ha residido en un país extranjero durante más de un año independientemente de las causas de su traslado, voluntario o involuntario, o de los medios utilizados, legales u otros" (ONU, 2018).

En Chile la migración ha crecido exponencialmente en la última década. Según datos elaborados por el Instituto Nacional de Estadísticas (INE) y el Departamento de Extranjería y Migración (DEM) en el informe "Estimación de personas extranjeras residentes en Chile", al 31 de diciembre del año 2019 la población extranjera residente estimada ascendió a 1.492 .522 personas. La población migrante es diversa y heterogénea, y su procedencia en su mayoría corresponde a América Latina y el Caribe, concentrando las mayores entradas de personas provenientes de los siguientes países: Venezuela $(30,5 \%)$, Perú (15,8\%), Haití (12,5\%), Colombia (10,8\%) y Bolivia (8,0\%), los que representa al $77,6 \%$ del total de la población migrante residente en Chile (INE, 2020).

Las principales razones que subyacen a la migración son la falta de empleo o de condiciones mínimas de trabajo, carencias en el acceso a los servicios básicos y bajos estándares de vida en el país de origen. La migración entendida bajo estas características se enmarca dentro de lo que se conoce como migración económica o también denominada migración forzada (Gzesh, 2008) la cual, según la autora, guarda relación con la vulneración de derechos generada por los países de origen, quienes no han otorgado las condiciones para que sus ciudadanos puedan permanecer en sus comunidades.

En Chile, la población migrante tiende a concentrarse mayoritariamente en zonas urbanas más que rurales, ascendiendo a 95,2\% del total de los cuales un $64 \%$ lo hace en las áreas metropolitanas de la región de Valparaíso, del Biobío y Metropolitana (Aninat y Vergara, 2019). Aún, cuando la población migrante participa activamente de la vida económica y social del país de acogida, tiende a concentrarse en grupos de pares pertenecientes a sus países de origen, propiciando la mantención de su propia cultura.

Según Tijoux (2013), la población extranjera que migra hacia Chile es posible categorizarla en dos grupos. La primera es la población catalogada como "extranjera" 
proveniente mayoritariamente de Europa y EEUU, los cuales, por sus condiciones económicas, redes de apoyo social y el ser "blancos" se radican en comunas de alto poder adquisitivo y de situaciones socioeconómicas favorables. El segundo grupo corresponde a población migrante que provienen desde América Latina y el Caribe, quienes lo hacen a sectores más desfavorecidos, que no poseen un alto nivel socioeconómico y se caracterizan por tener altos índices de vulnerabilidad económica y social. En concordancia con este postulado, los datos actuales indican que la población extranjera que migra desde América Latina reside en su mayoría en la comuna de Santiago o comunas aledañas como Independencia, Estación Central y Recoleta, quienes concentran la mayor cantidad de migrantes en el área metropolitana (Aninat y Vergara, 2019), o también en la periferia de la región Metropolitana, concentrando la mayor población en Quilicura, y de forma más marginal en Puente Alto o San Bernardo (INE, 2019).

¿Qué ocurre cuándo se migra a territorios en Chile en donde se concentran altos índices de pobreza y vulnerabilidad?

Tanto la migración como el desplazamiento forzado, se ven acompañados de una experiencia de desarraigo y vulnerabilidad. Se deja atrás a familias y amigos, se produce una ruptura con el núcleo de origen y se rompen espacios de identidad y relaciones, lo que inevitablemente provoca en un comienzo un choque cultural y posteriormente un sentimiento de extrañeza ante la nueva realidad (Ober et al., citado en Checa et al., 2007). No siempre la premisa de que la vida en el país de residencia será mejor que en el país de origen se cumple, lo cual es una contradicción con la idea central del fenómeno migratorio: sentirse plenamente incluidos en la sociedad de acogida y mejorar la calidad de vida.

Se entiende por vulnerabilidad como "algún tipo de amenaza, sean eventos de origen físico como sequías, terremotos, inundaciones o enfermedades, o amenazas antropogénicas como contaminación, accidentes, hambrunas o pérdida del empleo" (Ruiz, 2012, p. 64). Así también se comprende como una amenaza particular en relación a la salud, el ingreso y las capacidades que posean las personas de sobrevivir a cierto tipo de amenazas. Se refiere además a las "características de una persona o grupo y su situación, que influencian su capacidad de anticipar, lidiar, resistir y recuperarse del impacto de una amenaza" (Wisner et al., 2004, citado en Ruiz, 2012, p. 65). Además se comprende desde la intensidad del peligro al cual un grupo determinado se encuentra expuesto y la relación objetiva que poseen esos grupos en relación a sus propias condiciones materiales para salvaguardarse.

En ese contexto, la Comisión Económica para América Latina y el Caribe, CEPAL, a partir del estudio "La inmigración en Chile, una mirada multidimensional del año 2018", pone de manifiesto que se han cambiado las mediciones de pobreza desde el año 2013, a fin de poder medirla y categorizarla desde su multidimensionalidad, en base a criterios de vivienda, trabajo, salud y educación. La denominada pobreza multidimensional "consiste en medir directamente distintas carencias que pueden afectar a los miembros del hogar y que los califican como pobres cuando esas carencias, pondera- das, sobrepasan un cierto umbral" (Aninat y Vergara, 2019, p. 287).

El estudio muestra que al comparar la pobreza local con la de la población del migrante, existe una diferencia significativa en la medición del año 2017, la cual declara que la situación de pobreza de la población migrante es mucho peor que la de la población local. Esto se explica debido a que la población migrante de bajos ingresos ha aumentado en relación con la población local, afectando en gran medida a la población migrante provenientes de Bolivia, Haití y Colombia. En consecuencia, la población migrante que se 
encuentra arraigada a sus propios grupos de pares en contextos de vulnerabilidad, segregación y exclusión, pueden ser caracterizadas desde el concepto de "ciudadanía precaria” (Durán-Migliardi y Thayer-Correa, 2019, p. 114), la cual permite comprender la existencia de grupos de migrantes segregados y excluidos poseen dificultades mayores de inclusión en el país de acogida desde un punto de visa de incorporación precaria en el territorio en cuestión a nivel normativo, político y de interacción.

Por lo tanto, cuando la población migrante se incorpora a contextos altamente vulnerables, reproduce la marginalidad que en esos planos se desarrollan (Castel, 2009). La población vulnerable que se encuentra en la base de la escala social no siempre es tomada en cuenta para satisfacer las necesidades básicas de salud, educación y calidad de trabajo, que son parte indispensable de la convivencia en la sociedad de acogida con el fin de poseer oportunidades concretas para su plena inclusión, lo que al fin de cuentas van produciendo marginalidad y segregación.

De esta manera, todos los problemas que pueden afectar directa o indirectamente a la población migrante pueden ser consideradas como amenazas de exclusión, segregación y vulnerabilidad. Como ejemplo, Tijoux (2013) concluye que, en el caso específico de la inclusión de estudiantes peruanos, su nacionalidad es considerada como un aspecto negativo en contextos socioeconómicos vulnerables, lo que provocaría barreras inclusivas que afectan sus procesos de sociabilización y aprendizaje, siendo un grupo segregado y estigmatizado.

En consecuencia, migrar hacia un país distinto, el cual posee una cultura diferente al país de acogida, ya es un tema dificultoso, y lo es aún más cuando se migra a un contexto de pobreza y vulnerabilidad. En ese contexto, la construcción de redes de apoyo se desarrolla mayoritariamente con personas de la misma nacionalidad de origen, lo cual dificulta la inclusión social de las familias en el país de acogida tanto en términos sociales, laborales y de acceso a servicios básicos. El ámbito educativo también estaría siendo permeada por esta misma racionalidad, lo cual implica trabas en el proceso de inclusión educativa de estudiantes migrantes que serán analizadas a continuación.

\section{Migración en el plano educativo}

A partir de los datos entregados por el informe "Estimación de personas extranjeras residentes en Chile", al 31 de diciembre del año 2019, los menores de 15 años que migran junto a familiares también han crecido. En relación a personas en edad escolar, se declara que cada país en específico posee un alto porcentaje de menores de 15 años, vale decir en edad de ser escolarizados. Bolivia posee la cantidad mayor con un 13,7\%, seguido de Venezuela con un 12,5\%, Colombia con un $9,7 \%$, Argentina con un 7,2\% y Haití con un $5,6 \%$ (MINEDUC, 2018).

Según el Ministerio de Educación (2018), los estudiantes migrantes aumentaron entre el año 2015 (1,5\%) y el año 2017 (3,5\%). Los estudiantes extranjeros en su mayoría (57,5\%) se concentran en la educación municipal, en establecimientos particulares subvencionados el 33,1\%, en establecimientos particulares pagados el 7,9\% y el 1,5\% en Centros de Administración Delegada (CAD). Los datos anteriormente presentados indican que las escuelas se vuelven día a día más diversas y heterogéneas, lo que provoca que los Proyectos Educativos Institucionales (PIE) que se encontraban permeados por la realidad local, han debido adaptarse y transformarse de acuerdo a las nuevas realidades 
socioculturales que permitan la plena inclusión de estudiantes migrantes en el sistema educativo chileno.

Con la finalidad de favorecer la inclusión socioeducativa de niños, niñas y jóvenes en el sistema educativo regular, y en coherencia con la Ley General de Educación (2009), el año 2015 se promulga la Ley 20.845 de Inclusión Escolar (Ley de Inclusión Escolar, 2015), la cual tiene por objeto eliminar el financiamiento compartido -copago-, prohibir el lucro en establecimientos educacionales que reciben aportes del estado y regular la admisión de los y las estudiantes a partir de procesos de selección arbitrarios. Específicamente, la selección escolar y la migración tenían caminos paralelos, pero que bajo esta nueva óptica no podían continuar en esa dirección. Los establecimientos educacionales al no seleccionar al estudiantado, promueven en sí mismos la diversidad y la inclusión socioeducativa, ya que el sistema de admisión al ser aleatorio, provoca que todo estudiante pueda participar del algún establecimiento educacional sin importar su condición socioeconómica, cultural, étnica, de género, de nacionalidad o religión (Ley de Inclusión Escolar, 2015).

En la misma línea, la "Política nacional de estudiantes extranjeros 2018-2022" (MINEDUC, 2018), articula las acciones de inclusión educativa para la población migrante desde diversos enfoques, de los cuales destacan el Enfoque de Derechos, el de Inclusión, el de Género y el de Interculturalidad, entre otros; los cuales permitirán en concordancia con la política pública vigente la plena inclusión socioeducativa de los y las estudiantes migrantes.

Variadas investigaciones en Chile dan cuenta del fenómeno migratorio y las consecuencias que ésta ha tenido en los establecimientos educaciones, y por consiguiente en los procesos de enseñanza-aprendizaje. En el ámbito de la gestión educativa, respecto a lo que directoras y directores de escuela realizan a fin de favorecer la inclusión de niñas, niños y jóvenes migrantes, Quiroga y Aravena (2018) señalan que se deben desarrollar prácticas inclusivas en los establecimientos educacionales que incorporen a las familias del estudiante migrante con el fin de establecer relaciones de confianza y colaboración, que posibiliten "el no proyectar prejuicios socioeconómicos y culturales, especialmente, con estudiantes en condiciones de pobreza, inmigrantes o con dificultades cognitivas" (p. 104). Además, afirman que se debe contemplar el desarrollo profesional de directoras y directores de escuela en el ámbito de la planificación y análisis de resultados de los y las estudiantes, que permitan el diseño de prácticas inclusivas orientadas hacia las necesidades del estudiantado.

En el ámbito de la acción didáctica, Hernández (2016) declara que para abordar de buena manera los procesos educativos, parte de los problemas a considerar guardan relación con los procesos de formación inicial homogeneizadores y la implementación de un currículum descontextualizado. En ese sentido establece que los profesores y profesoras realizan procesos de integración curricular en el ámbito de asignaturas específicas como Historia y Geografía y Educación Musical, sin abordar para estos fines todas las asignaturas propuestas en el currículum nacional de enseñanza. Dentro de sus conclusiones se pueden destacar:

Las escuelas en contextos de alumnado migrante no han logrado implementar una política inclusiva que facilite la integración de los niños y niñas extranjeros. Se evidencia la ausencia de un plan y actividades concretas para eliminar la discriminación, el racismo y la xenofobia. (Hernández, 2016, p. 167) 
Por lo tanto, se puede esclarecer que una de las grandes problemáticas que poseen las escuelas a la hora de llevar a cabo procesos inclusivos de estudiantes migrantes guardan relación con aspectos curriculares y metodológicos, que pertmitan desarrollar acciones didácticas contextualizadas que consideren las particularidades de los y las estudiantes, con el fin de sobrepasar la barrera de la integración escolar para convertirse finalmente en escuelas inclusivas. En la misma dirección Poblete (2018) destaca que:

\begin{abstract}
Las escuelas y los docentes realizan esfuerzos tendientes a visibilizar la cultura de los niños y niñas extranjeros, pero que, en ese proceso, enfrentan dos dificultades: por un lado, la poca flexibilidad del currículo (al menos asi es percibido en las escuelas y ratificado por algunos estudios); y, por otro, la falta de preparación de los profesores para llevar a cabo estas adaptaciones, toda vez que ellos mismos indican que no cuentan con las herramientas para hacerlo. (p. 61)
\end{abstract}

Así también, los y las docentes "poseen imaginarios que tienden a atribuir a los y las migrantes una serie de déficits académicos y de comportamiento que dificultan su proceso de inclusión y su plena participación en las escuelas" (Cerón et al., 2017, p. 242), las cuales son representaciones orientadas a la discriminación y la exclusión de un grupo en particular, imposibilitando la plena inclusión socioeducativa del estudiante migrante.

En consecuencia, lo que Chile ha intentado desarrollar como prácticas pedagógicas inclusivas que consideren a estudiantes migrantes son las que intentan relevar su cultura de origen, orientando la acción didáctica en asignaturas que facilitan el trabajo de los y las profesores/as y que son transversales a las diversas nacionalidades. Además, el profesorado en su conjunto no posee las herramientas necesarias para lograr la plena inclusión socioeducativa de sus estudiantes, ya que las prácticas pedagógicas que se creen inclusivas tienden a ser excluyentes. Esto último se evidencia desde un punto de vista didáctico curricular, puesto que las escuelas no han adaptado sus prácticas pedagógicas que considere las realidades de la nueva diversidad, sin realzarlas de manera individualizada, sino más bien procurando su resguardo, pero incentivando la construcción de una nueva realidad, de una nueva cultura inclusiva.

\title{
4. La educación inclusiva como respuesta a la diversidad
}

El desarrollo de prácticas socioeducativas desde un Enfoque Inclusivo al interior de los establecimientos educacionales, no han estado exentas de dificultades, respecto a su comprensión y maneras de operar. Se hace necesario entonces entenderla desde su concepción global, su epistemología, y cómo ha incidido en el desarrollo de políticas públicas en el ámbito nacional.

En el plano internacional, la UNESCO (2008) define la educación inclusiva como:

Un proceso de fortalecimiento de la capacidad del sistema educativo para atender a todos los educandos. Por consiguiente, es un principio general que debería guiar todas las políticas y prácticas educativas, partiendo de la convicción de que la educación es un derecho humano fundamental y el fundamento de una sociedad más justa. (p. 12)

La educación inclusiva, al estar orientada desde un Enfoque de Derechos, brinda la posibilidad de enaltecer la acción educativa desde un marco jurídico potente e innegociable, que permita el aprendizaje de los y las estudiantes desde su propia particularidad, sin hacer distinciones arbitrarias o promoviendo prácticas segregadoras y excluyentes. 
En Chile, la educación inclusiva ha estado ligada durante todo su desarrollo sociohistórico y epistemológico a la atención de personas que presentan necesidades educativas especiales y/o en situación de discapacidad, dependiendo del enfoque subyacente al momento histórico de la Educación Diferencial (Godoy et al., 2004), la cual tuvo un cambio de orientación desde el momento de aprobar la Ley de Inclusión Escolar (Ley de Inclusión Escolar, 2015). En ese sentido,

\section{La Educación Inclusiva supone un cambio de enfoque en la discusión, la cual implica dejar de diseñar acciones para un otro diferente que tiene cabida sólo en espacios de la sociedad previamente establecidos, que estarían dentro de una mirada de Integración Escolar, sino más bien posibilitando la coexistencia y participación de cada individuo de lleno en cada esfera de la sociedad. (Román Soto, 2019, p. 11)}

Por lo tanto, el foco de la discusión deja de estar centrada en el déficit que se realiza desde la óptica de la integración escolar pasando a relevar la diversidad y diferencias de los y las estudiantes en contextos educativos también diversos propiciando el desarrollo de una nueva cultura inclusiva.

Para la comprensión de la diversidad como fenómeno y conceptualización, el MINEDUC (2017) la entiende como parte de las historias de vida de los sujetos participantes del proceso de enseñanza-aprendizaje escolar, la cual debe ser considerada al momento de la realización acciones didácticas. La experiencia de vida y los aprendizajes previos pasan a ser cruciales en este proceso, los cuales servirán de punta pie inicial para que el proceso de enseñanza-aprendizaje sea exitoso y contextualizado.

El constructivismo radical también ha aportado en la construcción de este concepto. Maturana (1990) promueve el concepto de diversidad desde la premisa de aceptarnos como otros distintos y legítimos en contextos de convivencia diversos. Es así como la distinción de la diversidad se realiza desde una concepción biológica, puesto que nuestra diferenciación entre individuos nos permite comprender que cada uno entiende la realidad a partir de su propia experiencia, subjetividad y cotidianeidad.

El autor sugiere dos caminos explicativos, la objetividad y la objetividad entre paréntesis (Maturana, 2013). En el camino de la objetividad, la realidad ocurre independiente del individuo, existiendo un solo dominio explicativo. Por tanto, se vive en las certezas y en la constante negación del otro como un legítimo. En cambio, en la (objetividad), la realidad no ocurre independiente del sujeto, puesto que biológicamente somos diferentes, nuestras experiencias e historias de vida son diferentes y, en consecuencia, interactuamos con el mundo de manera particular.

En ese sentido, la diversidad entendida como un "proceso constructivo que depende de la historia de interacciones en las que participa el observador, que emerge en el mismo momento en que distingue el mundo que produce" (Druker, 2020, p. 237), nos permite avanzar hacia la comprensión de este nuevo camino explicativo, en donde lo crucial radica en la interacción con otros y con el mundo, a fin de constituirnos como seres legítimos. De igual modo, Ibáñez et al. (2012) conciben la diversidad como:

La consecuencia de modos distintos de construir significados que dan lugar a una visión de mundo diversa en algunos o en muchos sentidos, no mejor o peor sino sólo diferente, que se constituye en el lenguaje según el modo de convivencia propio de cada cultura o subcultura. (p. 217)

La educación inclusiva al poner su énfasis en la diversidad de cada individuo, promueve la construcción de una nueva escuela, que considera las diferencias y particularidades de los 
participantes. Su atención está centrada en la historia de cada estudiante, sus contextos socioculturales y experiencias previas, en climas de igualdad y aceptación mutua. Promueve además el desarrollo de espacios socioeducativos diversos y diversificados, que permitan disminuir las brechas de desigualdad existentes hacia grupos excluidos con la finalidad de incorporar a todas y todos en base al respeto mutuo.

En concordancia con la educación inclusiva y durante el mismo año de la aprobación de la Ley de Inclusión Escolar, Chile aprueba el año 2015 el Decreto de Ley n ${ }^{\circ}$ 83, el cual "aprueba criterios y orientaciones de adecuación curricular para estudiantes con necesidades educativas especiales de educación de párvulos y educación básica” (Decreto de Ley $\left.n^{\circ} 83,2015\right)$. Este se basa en el principio de diversidad, y su accionar apunta más allá de las Necesidades Educativas Especiales, puesto que se busca:

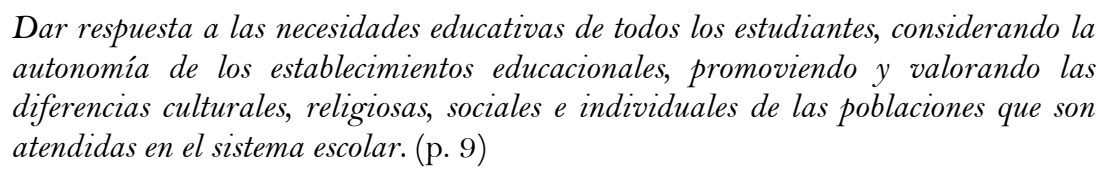

Dar respuesta a las necesidades educativas de todos los estudiantes, considerando la autonomía de los establecimientos educacionales, promoviendo y valorando las diferencias culturales, religiosas, sociales e individuales de las poblaciones que son atendidas en el sistema escolar. (p. 9)

Además, estipula cuatro principios orientadores para la toma de decisiones de la adecuación curricular: Igualdad de oportunidades, calidad educativa con equidad, inclusión educativa y valoración de la diversidad, y flexibilidad en la respuesta educativa. En ese sentido, releva el principio de diversidad con el fin de realizar acciones didácticas inclusivas para estudiantes marginados o en riesgo de ser excluidos del sistema escolar, valorando sus diferencias, reconociéndolas, respetándolas, y propiciando el acceso y la participación de todas y todos en las diversas unidades educativas.

Para tales efectos, el aula también debe ser reconceptualizada. Para lograr una inclusión educativa plena y efectiva, no basta con que se generen las condiciones declaradas por el marco normativo, sino que el aula debiese ser entendida como:

\begin{abstract}
Un lugar de convivencia democrática, que valora la diversidad de cada uno de los sujetos que la componen; en dónde estudiantes y profesores construyen realidades a partir de sus historias de vida y sus emociones, permitiéndoles consensuar acciones didácticas organizadas, pertinentes y adecuadas, que promueven la inclusión sociocultural y posibilitan el aprendizaje de todas y todos. (Román Soto, 2019, p. 25)
\end{abstract}

El espacio didáctico es concebido entonces como un lugar de encuentro y co-construcción de aprendizajes, el cual es un elemento fundamental a la hora de desarrollar prácticas inclusivas que considere al estudiante migrante. La escuela no debe únicamente adaptarse a la nueva realidad, sino también transformarse, y es en ese proceso que los postulados que subyacen a modelos de educación intercultural toman fuerza y proyección, ya que se encuentran en la misma racionalidad que la educación inclusiva, y permitirán que la escuela se comprenda desde sus diferencias y valore la diversidad como eje transversal a la acción didáctica y socioeducativa.

\title{
5. La interculturalidad, una nueva racionalidad para la construcción y aceptación de la diversidad en el espacio didáctico
}

El fenómeno migratorio, ha desembocado en nuevos cuestionamientos que realizan establecimientos educacionales a la hora de abordar la nueva realidad multicultural que se 
vive dentro del espacio escolar. Esto ha provocado contradicciones constantes en el profesorado y cuerpos directivos para enfrentar con éxito el fenómeno migratorio y la nueva escuela que se intenta construir.

Para el sentido común del profesorado hablar de multiculturalidad en el espacio didáctico y su diversidad, promoviendo la inclusión educativa desde acciones enmarcadas en la Integración Escolar, que conceptualiza la realidad desde el déficit y/o la diferencia, tiende a la confusión de conceptos y miradas epistemológicas, en donde se intenta realzar cada una de las culturas que se encuentran presentes dentro del espacio didáctico, sin comprender el modelo de exclusión que se intenta consolidar.

En ese sentido, se hace necesario comprender la racionalidad didáctico-curricular que operan en los discursos educativos, con el fin de aterrizar las diferentes concepciones epistemológicas que se tienen respecto a lo educativo y su afectación en el desarrollo de las prácticas pedagógicas dentro del espacio didáctico.

El enfoque Crítico del currículum, tensiona al poder desde su concepción más profunda, lo observa como modelo de dominación, reproducción cultural y violencia simbólica (Bourdieu, 1970). Cuestiona al capitalismo, al neoliberalismo y a la educación de clases desde prácticas educativas transformativas, emancipadoras y de resistencia, velando por el surgimiento del oprimido frente y contra los poderosos, dejando atrás el modelo educativo tradicional, que da cuenta de elementos rígidos y pragmáticos como lo son la enseñanza, el aprendizaje, la metodología y la evaluación (Da Silva, 1999). El modelo poscrítico del currículum orientará su quehacer en dirección de la construcción de identidades, relevando la diferencia y la subjetividad, el cual propondrá "nuevas temáticas, tales como diversidad, inclusión, interculturalidad, género, demandas identitarias y culturales en el contexto educativo del currículum escolar" (Aranda, 2017, p. 5), que permitirán reconfigurar la escuela y el espacio didáctico.

En ese contexto, el fenómeno multicultural que se vive en el espacio didáctico a partir de la migración, puede definirse desde una postura crítica a partir de su nacimiento en culturas occidentales "para caracterizar la situación diversa e indicar la existencia de múltiples culturas en un determinado lugar planteando así su reconocimiento, tolerancia y respeto" (Walsh, 2008, p. 140). Este término perpetúa la desigualdad e inequidad social por cuanto su uso determina y orienta las políticas públicas al servicio del modelo neoliberal en concordancia con una visión transnacional dentro del mercado mundial.

Por lo tanto, la multiculturalidad invita a la comprensión que diversas culturas puedan convivir dentro de un mismo territorio y contexto, procurando salvaguardar sus diferencias individuales al servicio de la política neoliberal, sin dejar que existan nuevas prácticas asociadas a procesos inclusivos, que sin duda ponen en jaque al poder, puesto que la creación espontánea y colaborativa de nuevas sociedades delimita su campo de acción como agente de dominación ideológica. Para la autora, en cambio, la interculturalidad es una concepción en construcción aún inexistente "Va mucho más allá del respeto, la tolerancia y el reconocimiento de la diversidad; señala y alienta, más bien, un proceso y proyecto social político dirigido a la construcción de sociedades, relaciones y condiciones de vida nuevas y distintas" (Walsh, 2008, p. 140).

En este camino de construcción y transformación sociocultural, cuando en la actualidad hablamos de Interculturalidad en la educación chilena, se deja atrás las concepciones primarias del término que ponían énfasis en los procesos de inclusión socioeducativa de 
pueblos originarios, las cuales amparadas en la Ley Indígena 19.253 de 1993, promueve el "desarrollo de un sistema de educación intercultural bilingüe con la finalidad de preparar a los educandos indígenas para desenvolverse en forma adecuada tanto en su sociedad de origen como en la sociedad global” (Ley Indígena, 1993). De este modo, la interculturalidad deja de estar arraigada al mundo indígena, así como la Inclusión Educativa a la educación especial, tal como lo indica Druker (2020), en donde ambos enfoques se operacionalizan "focalizadas en los diferentes y no en las diferencias, evidenciando una construcción vertical del otro, que lo reduce a una diferencia conocida, predecible y siempre definida desde la mismidad" (p. 232).

En sus inicios, tanto la educación intercultural como la educación inclusiva estuvieron subscritas a modelos de compensación a partir del déficit, los cuales desde su evolución socio-histórica y epistemológica, han logrado desprenderse de esta racionalidad a partir de nuevas relaciones humanas que buscan la armonía y simetría entre los participantes del espacio escolar. No obstante, las relaciones de poder permeadas constantemente por el modelo neoliberal, permiten en la actualidad prácticas orientadas a la discriminación positiva de grupos excluidos, puesto que en concordancia con el constructivismo radical de Maturana (1990), la tolerancia sigue siendo una negación postergada del otro.

En consecuencia, tanto la interculturalidad como la educación inclusiva abren espacios para una nueva realidad de respeto, empatía y aceptación, que establece relaciones horizontales y recíprocas, en donde la escuela como institución juega un rol fundamental para efectos de concretar procesos inclusivos acordes a la nueva realidad multicultural.

En el plano internacional la UNESCO (2006) releva los procesos inclusivos de la población migrante proponiendo acciones coherentes con el Enfoque de Derechos y en concordancia con lo estipulado por la educación intercultural, la cual promueve la interrelación entre cultura y educación, lenguaje, religión y sus prácticas asociadas desde el respeto y fomento de la diversidad desde un Enfoque Intercultural a partir de tres principios:

- Principio I: La educación intercultural respeta la identidad cultural del educando impartiendo a todos, una educación de calidad que se adecue y adapte a su cultura.

- Principio II: La educación intercultural enseña a cada educando los conocimientos, las actitudes y las competencias culturales necesarias para que pueda participar plena y activamente en la sociedad.

- Principio III: La educación intercultural enseña a todos los educandos los conocimientos, actitudes y las competencias culturales que les permiten contribuir al respeto, el entendimiento y la solidaridad entre individuos, entre grupos étnicos, sociales, culturales y religiosos y entre naciones.

Estos principios orientarán la práctica socioeducativa en el espacio didáctico, respetando y valorizando la diversidad cultural (Mardones, 2017), intencionado una educación intercultural que considere la identidad cultural y las competencias culturales como elementos esenciales.

En lo que concierne a la migración como fenómeno actual, la "Política nacional de estudiantes extranjeros 2018-2022" (MINEDUC, 2018), pone énfasis en el Enfoque Intercultural, ya que: 
La interculturalidad es un horizonte social ético-político en construcción, que enfatiza relaciones horizontales entre las personas, grupos, pueblos, culturas, sociedades y con el Estado. Se sustenta, entre otros, en el diálogo desde la alteridad, facilitando una comprensión sistémica e histórica del presente de las personas, grupos y pueblos diversos que interactúan permanentemente en los distintos espacios territoriales. La interculturalidad favorece la creación de nuevas formas de convivencia ciudadana entre todos, sin distinción de nacionalidad u origen. Para ello, el diálogo simétrico es posible reconociendo y valorando la riqueza de la diversidad lingüistico-cultural, natural y espiritual. (p. 21)

Esta nueva escuela desde una concepción poscrítica y en relación a lo propuesto por una educación para la Justicia Social (Jiménez-Vargas et al., 2019), reflexiona en función de una nueva realidad dentro del espacio escolar, la cual permite la construcción de una ciudadanía crítica, activa, democrática y emancipada. La escuela ya no es el motor del cambio social, como la caracterizaban los autores críticos, sino que es el medio por el cual la sociedad se transformará en su conjunto, permitiendo que todo grupo sin importar su condición y/o distinción sea parte de la comunidad educativa y desarrolle un potente compromiso social.

Desde esta mirada social reconstruccionista, se nos invita a mirar la Educación Intercultural desde la Justicia social, la cual propone la reflexión bajo la premisa de cuatro elementos clave (Jiménez-Vargas y Montecinos-Sanhueza, 2019, p. 111):

- Situar a las familias y las comunidades dentro de un análisis de las desigualdades estructurales y, de esta forma, evitar explicaciones educativas basadas únicamente en características individuales de los estudiantes o a partir de su pertenencia cultural;

- Desarrollar relaciones de reciprocidad con estudiantes, sus familias y comunidades, para promover de esta forma relaciones más horizontales y con mayor grado de conocimiento mutuo entre docentes y estudiantes;

- Enseñar con altas expectativas, para superar las creencias asociadas al déficit cultural y desarrollar experiencias educativas estimulantes y motivantes para todos los estudiantes; finalmente,

- Crear un currículum inclusivo, que integre no solo el saber oficial sino las diferentes voces y perspectivas tradicionalmente silenciadas en el ámbito educativo.

En ese sentido, la interculturalidad en concordancia con la inclusión educativa, permite construir desde la propia diversidad existente en el espacio didáctico y escolar, prácticas socioeducativas coherentes, contextualizadas, que respeten y valoren la diversidad, ya no centradas en lo diferente sino en la diferencia de todas y todos. Desde este enfoque, la desigualdad observada en la escuela deja de estar centrada en el déficit individual o de colectivos excluidos, ya que es concebida como una problemática de la comunidad educativa en su totalidad.

Consecuentemente, la escuela como medio de transformación sociocultural, será el escenario en que todos los miembros de la comunidad educativa se sientan parte, con el fin de armonizar las relaciones humanas para no solo cuestionar al poder, sino corromperlo desde su estructura más arraigada, permitiendo arrancar toda forma de desigualdad y discriminación arbitraria. 
En definitiva, al interior del espacio didáctico, la creación de un currículum inclusivo bajo los supuestos de la diversificación de los procesos de enseñanza-aprendizaje, tal como lo indica el decreto $\mathrm{n}^{\circ} 83$ que tiene como objetivo "maximizar las oportunidades de aprendizaje de todos los estudiantes, considerando la amplia gama de habilidades, estilos de aprendizaje y preferencias" (Decreto de Ley $\mathrm{n}^{\circ}$ 83, 2015), permitirán lograr lo propuesto por la Educación Intercultural y Educación Inclusiva. Este decreto, considera tres principios fundamentales bajo el alero del Diseño Universal de Aprendizajes (DUA): proporcionar múltiples medios de presentación y representación, proporcionar múltiples medios de ejecución y expresión, y proporcionar múltiples medios de participación y compromiso; los cuales permitirán y promoverán la creación de una nueva escuela a partir de la convivencia democrática y procesos dialógicos pertinentes y adecuados de transformación y emancipación.

\section{Conclusiones}

La política pública actual de inclusión educativa, cobra un nuevo sentido más allá de sus primeros postulados que orientaban su quehacer educativo a estudiantes que presentan necesidades educativas especiales. Su foco actual, tanto normativo como epistemológico intenta, tanto dentro como fuera del espacio didáctico, valorizar y comprender la diversidad desde diferentes postulados y aproximaciones. En coherencia con una mirada poscrítica de lo educativo, la educación inclusiva e intercultural intentan brindar a la escuela nuevas perspectivas de reflexión y construcción, las cuales permitirán a las comunidades educativas transformaciones dialógicas e inclusivas para la creación de una nueva realidad, de aceptación y respeto por la diferencia en contextos de migración y vulnerabilidad.

Migrar a un país extranjero es difícil, y más aún lo es el hacerlo en contextos de vulnerabilidad. Los procesos de desigualdad que desde ahí se desprenden podrían contenerse si la escuela no fuese solo un lugar de transmisión de conocimientos, o de desarrollo de habilidades y actitudes, sino más bien un lugar de encuentro, contención, aprendizaje y transformación. Para lograr insertarse y permanecer en país de acogida el rol de la escuela es fundamental, ya que permitirá no solo que el estudiante participe de procesos inclusivos exitosos en la comunidad educativa, sino también que sus familias sean parte de dicho proceso. En ese sentido, tanto la interculturalidad como la inclusión educativa pueden desarrollarse plenamente en las instituciones escolares como enfoques complementarios, el primero desde la construcción de comunidades escolares más justas, y el segundo desde la valoración de la diversidad desde la mirada de la justicia social tanto el desarrollo de prácticas pedagógicas, como en la facilitación de procesos inclusivos para las familias de las y los estudiantes extranjeros.

En ese sentido, el espacio educativo permite trabajar de manera mancomunada entre todos los integrantes de la comunidad escolar, asegurando el derecho que poseen, niños, niñas y jóvenes, sin importar su origen y condición política, social y/o económica, de desarrollarse como seres libres y autónomos en esta nueva sociedad, de características dinámicas y que se encuentra en constante transformación, con el fin de recibir una educación pertinente, adecuada y contextualizada, propias de una mirada inclusiva.

Así mismo, la interculturalidad, en tanto enfoque, asegura que los estudiantes migrantes sin distinciones arbitrarias y segregadoras, puedan participar de ambientes educativos de 
aceptación y colaboración, propiciando la interacción de los participantes en contextos diversos. Así la escuela, desde una racionalidad inclusiva e intercultural, nos permite encaminarnos a reflexiones propias de la Justicia Social, la cual apoya la creación de una nueva escuela desde la horizontalidad y la no discriminación, en donde las problemáticas son de la comunidad y no en particular de los grupos excluidos a priori.

Finalmente, para lograr este tipo de reflexiones en las comunidades educativas, se propone que las escuelas desarrollen acciones didácticas y prácticas inclusivas desde la construcción de conocimiento basados en la Investigación-Acción, la cual se encuentra dentro del paradigma Crítico Emancipador (Latorre, 2005), puesto que incorpora ideas de la teoría crítica, la cual postula un avance desde la concepción técnica del currículum hacia una reflexión constante y transformativa del profesorado.

El paradigma Crítico Emancipador, en coherencia con la Investigación-Acción, pone su foco en la praxis de los y las profesores/as y lo articula con el contexto sociocultural en el cual se encuentran inmersos. Además, al ser un proceso crítico de reflexión y comprensión, permite la construcción de conocimiento proponiendo alternativas en la acción en base a la práctica y reflexión pedagógica en torno a la acción y transformación de las acciones socioeducativas individuales y colectivas en climas de colaboración.

\section{Agradecimientos}

Agradezco al Doctorado en Educación de la Universidad Metropolitana de Ciencias de la Educación que, mediante su "Programa de becas doctorales 2019", se hizo partícipe de este estudio.

\section{Referencias}

Aranda, V. (2017). Inmersión en el sistema escolar para el aprendizaje contextualizado del pensamiento crítico y post-crítico en educación. Diálogos Educativos, 18(33), 4-19.

Aninat, I. y Vergara, R, (2019). Inmigración en Chile. Una mirada Multidimensional. Centro de Estudios Públicos.

Bourdieu, P (1970). La réproduction. Editions de minuit.

Castel, R. (2009). La montée des incertitudes. Editions du seuil.

Cerón, L., Pérez, M. y Poblete, R. (2017). Percepciones docentes en torno a la presencia de niños y niñas migrantes en escuelas de Santiago: Retos y desafíos para la inclusión. Revista Latinoamericana de Educación Inclusiva, 1 1(2), 233-246

Checa, F., Arjona, A. y Checa, J. (2007). El extrañamiento cultural en espacios migratorios La juventud andaluza ante el reto de la multiculturalidad. Migraciones Internacionales, 4(1), 111 140 .

Da Silva, T. (1999). Documentos de identidad. Una introducción a las teorías del currículo. Editorial Auténtica.

Druker, S. (2020). El giro epistemológico: De la diversidad de los otros a la diversidad como condición del encuentro. Revista de Estudios y Experiencias en Educación, 19(39), 227-239

Durán-Migliardi, C. y Thayer-Correa L. (2020). Ciudadanía precaria: Hacia una definición conceptual para la caracterización de los procesos migratorios contemporáneos. Republicana, 28, 97-117. https://doi.org/10.21017/rev.repub.2020.v28.a78 
Godoy, P., Meza, M. y Salazar, A. (2004). Antecedentes históricos, presente y futuro de la educación especial en Chile. Ministerio de Educación.

Gzesh, S. (2008). Una redefinición de la migración forzosa con base en los derechos humanos. Migración y Desarrollo, 10, 97-126.

Hernández, A. (2016). El currículo en contextos de estudiantes migrantes: Las complejidades del desarrollo curricular desde la perspectiva de los docentes de aula. Estudios Pedagógicos, 27(2), 151-169.

Ibáñez N., Díaz, T., Druker, S. y Rodríguez M. S. (2012). La comprensión de la diversidad en interculturalidad y educación. Convergencia, Revista de Ciencias Sociales, 19(59), 215-240.

Jiménez-Vargas, F. y Montecinos-Sanhueza, C. (2019). Polifonía en educación multicultural: enfoques académicos sobre diversidad y escuela. Magis. Revista Internacional de Investigación en Educación, 12(24), 105-128. https://doi.org/10.11144/Javeriana.m12-24.peme

Instituto Nacional de Estadísticas y el Departamento de Extranjería y Migración. (2020). Memoria anual migración en chile, 2019. INE.

Latorre, A. (2005). La investigación-acción. Conocer y cambiar la práctica educativa. Edición Graó.

Mardones, T. (2017). Educación intercultural en el currículum nacional chileno. Revista Intersecciones Educativas, 7(1), 69-84.

Maturana, H. (1990). Emociones y lenguaje en educación y política. JC Saez Editor.

Maturana, H. (2013) La objetividad. Un argumento para obligar. Granica.

Ministerio de Educación. (2017). Informe nacional de la calidad de la educación 2017. Los desafíos de educar para la inclusión y la diversidad. Ministerio de Educación, Chile.

Ministerio de Educación. (2018). Política nacional de estudiantes extranjeros 2018-2022. Ministerio de Educación.

Organización de Naciones Unidas. (2019). Noticias ONU. ONU.

Poblete, R. (2018). El trabajo con la diversidad desde el currículo en la escuela con presencia de niños y niñas migrantes: Estudio de casos en escuelas de Santiago de Chile. Perfiles Educativos, 40(159), 51-65.

Quiroga M. y Aravena, F. (2018) La respuesta de directores escolares ante la respuesta de la inclusión educativa en Chile. Revista Calidad en la Educación, 49, 82-111.

Román Soto, D. (2019). La educación inclusiva en Chile. Tensiones y encuentros entre la política pública vigente (Decreto no170, 2009 y Decreto no83, 2015) y el espacio didáctico. Revista Akadèmeia, 18, 88-118.

Ruiz, N. (2012). La definición y medición de la vulnerabilidad social. Un enfoque normativo. Investigaciones Geográficas, Boletín del Instituto de Geografía, 77, 63-74.

Tijoux, M. (2013). La escuela de la inmigración en la ciudad de Santiago: Elementos para una educación contra el racismo. Polis, 12(35), 287-307.

UNESCO. (2006). Directrices de la UNESCO sobre educación intercultural. UNESCO.

UNESCO. (2008). La educación inclusiva: El camino hacia el futuro. UNESCO.

Walsh, C. (2008). Interculturalidad, plurinacionalidad y decolonialidad: Las insurgencias políticoepistémicas de refundar el Estado. Tabula Rasa, 9, 131-152. 


\section{Breve CV del autor}

\section{David Román Soto}

Profesor de Educación Diferencial en Problemas de Aprendizaje, Universidad Metropolitana de Ciencias de la Educación (UMCE). Master en Trabajo Social y Políticas Públicas, Universidad de Fribourgo, Suiza. Becado del programa de Doctorado en Educación (UMCE). Académico del Departamento de Educación Diferencial UMCE. Docente de la carrera de Educación Diferencial, (UAHC). Líneas de Trabajo e Investigación: Educación Inclusiva, Migración, Interculturalidad, Didáctica y Formación Inicial Docente. Coordinador Académico Fundación Súmate por la Inclusión. ORCID ID: https://orcid.org/0000-0002-0075-4094. Email: david.roman@umce.cl 